\title{
Case Series-An Overview of Extracapsular Dissection of Parotid Tumours
}

\author{
Deepalakshmi T1, Rai A $^{2 *}$, Mahesh SG ${ }^{3 *}$, Devan \\ $\mathbf{P P}^{4 *}$, Bhandary $\mathbf{R}^{1 *}$ and Pai $\mathbf{V}^{5 *}$ \\ ${ }^{1}$ Assistant Professor, Department of ENT, AJ IMS, \\ Mangalore, Karnataka, India \\ 2Post-Graduate, Department of ENT, AJ IMS, Mangalore, \\ Karnataka, India \\ ${ }^{3}$ Professor and Head of the Department, Department of \\ ENT, AJ IMS, Mangalore, Karnataka, India \\ 4Professor, Department of ENT, AJ IMS, Mangalore, \\ Karnataka, India \\ ${ }^{5}$ Senior Resident, Department of ENT, AJ IMS, \\ Mangalore, Karnataka, India \\ *Corresponding author: Anushree Rai, Post- \\ Graduate, Department of ENT, AJ IMS, Mangalore, \\ Karnataka, India
}

Mahesh SG, Professor and Head of the department, Department of ENT, AJ IMS, Mangalore, Karnataka, India

Devan PP, Professor, Department of ENT, AJ IMS,

Mangalore, Karnataka, India

Rukma Bhandary, Associate Professor, Department of ENT, AJ IMS, Mangalore, Karnataka, India

Vishwas Pai, Senior Resident, Department of ENT,

AJ IMS, Mangalore, Karnataka, India

Received: August 05, 2021; Accepted: September 01,

2021; Published: September 08, 2021

\section{Introduction}

Surgical treatment of benign parotid tumours has been debated for decades. ECD technique is conception ally different to the classical surgical approaches to parotid neoplasms, which are essentially a dissection of the facial nerve. In contrast, extra capsular dissection avoids a formal nerve dissection in preference to a careful dissection around the tumour itself. ECD involves a careful blunt dissection through the parotid tissue by way of a cruciate incision placed directly over the tumour. The neoplasm is then removed with a 2 to $3 \mathrm{~mm}$ rim of normal parotid parenchyma surrounding the tumour [1].

\section{Methods}

This is a descriptive study with 30 consecutive patients undergone ECD with the following variable took into our consideration; size of the tumor, side whether right or left, sex, age, time of the operation and complications. All the cases share the following; same operator, same surgical approach, which is, modified Blair incision, same closure technique, corrugated drain was used for all cases along with pressure dressing. All patients were discharged on the second postoperative day after removal of the corrugated drain. Indications for extra capsular dissection, The decision to do ECD of the parotid tumors can be made clinically; superficial, small, mobile with welldefined tumor margin and with no clinical evidence of malignant involvement such as facial nerve paralysis. These clinical signs give the surgeon initial assessment of the benign nature of the tumor

\section{Imaging}

Three features must be considered during imaging; nature, location and extent of the tumor. The first line imaging technique, cheapest, quickest and usually the only required modality to confirm the benign nature of the tumor and define these three features is the ultrasound. While MRI and/or CT is used if there is any doubt of the diagnosis such as a large tumor; MRI to exclude the deep lobe involvement or CT to exclude bony involvement, giving the threedimensional structure of the tumor and its relationship to the surrounding structures.

\section{Inclusion criteria}

Selection criteria only benign parotid tumor located unilaterally in the superficial part of the parotid was selected for the ECD. In this study, we discriminated benign from malignant tumors by history, clinical examination, FNA, ultrasound, MRI and/or CT scan [4].

\section{Exclusion criteria}

Tumors in the deep lobe, large $(>5 \mathrm{~cm})$ tumors in the superficial lobe and tumors with suspected malignancy as indicated clinically, by FNA, MRI and/or CT (central necrosis, irregular margins, infiltration of adjacent structures and the existence of any questionable cervical lymphadenopathy) were excluded. 


\section{Case Presentation}

\section{Case 1}

Warthin's Tumor: A 64-year-old male came with complaints of swelling in left side of neck since 1-year (Figure 1A). All necessary interventions were done. Computed tomography scan was done which revealed heterogeneous mass lesion, with entirely cystic masses with a peripheral solid enhancing rim. Extra capsular dissection of parotid tumor with facial nerve preservation was done under general anesthesia (Figure 1B) and specimen sent for histopathological examination (Figure 1C). Histopathology revealed- warthin's tumorencapsulated cystic tumor tissue with multiple papillary projections lined by two layers consisting of tall oxyphilic columnar cells and underlying discontinuous basal cells (Figure 2).

\section{Case 2}

Sialadenoma papilliferum of the parotid: A 47-year-old male patient came with complaints of painless swelling in the parotid region since 6 months (Figure $3 \mathrm{~A}$ ). Clinical examination revealed a well-defined, non -tender cystic swelling in the right parotid with intact overlying skin and no significant cervical lymphadenopathy. All hematological parameters were within normal limits. In fine needle aspiration, cytology- smears showed dispersed tiny irregular monolayered sheets of cells predominantly showing squamoid features of oncocytic cells with granular grey cytoplasm. Computed tomography-well defined thick walled predominantly cystic lesion with peripheral solid component in the superficial right parotid region. Extra capsular dissection of parotid tumor with facial nerve preservation was done under general anesthesia (Figure 3B). The

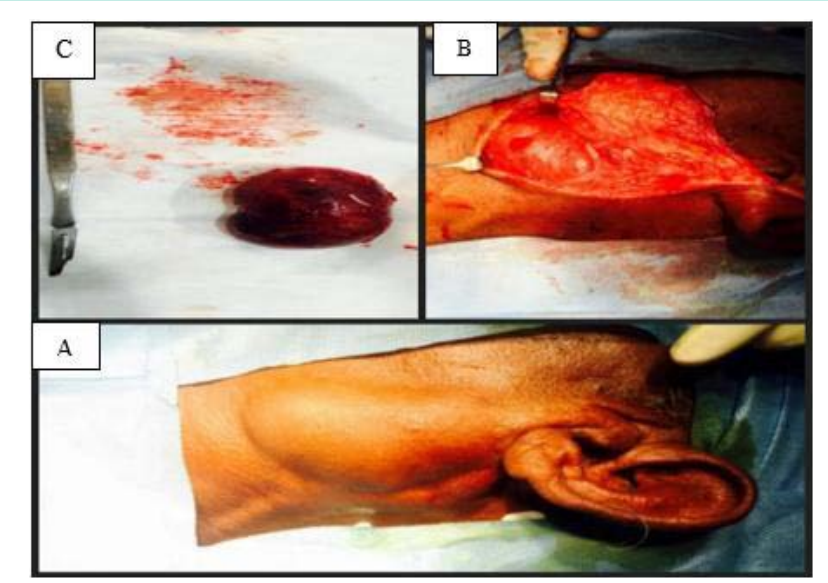

Figure 1: A) Swelling in left side of neck; B) Extracapsular dissection of parotid tumours with facial nerve preservation; C) Dissected specimen.

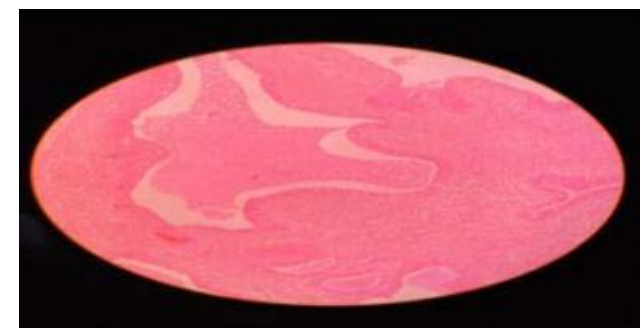

Figure 2: Histopathology revealed-warthin's tumor.

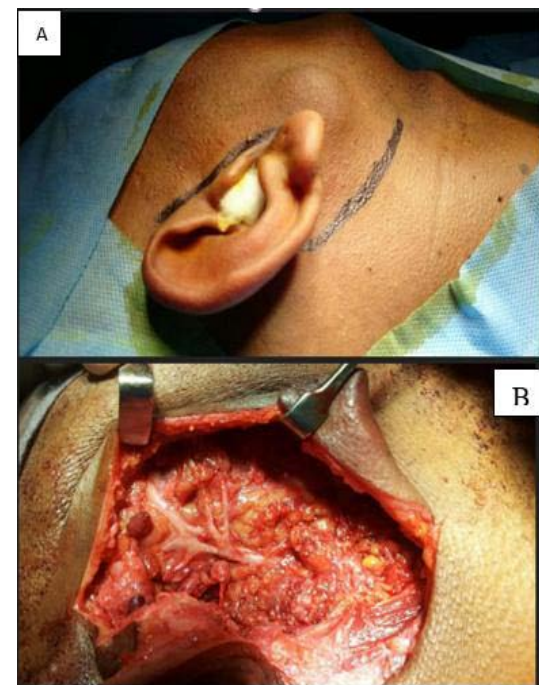

Figure 3: A) Swelling noted in the right parotid region; B) Extracapsular dissection of parotid tumours.

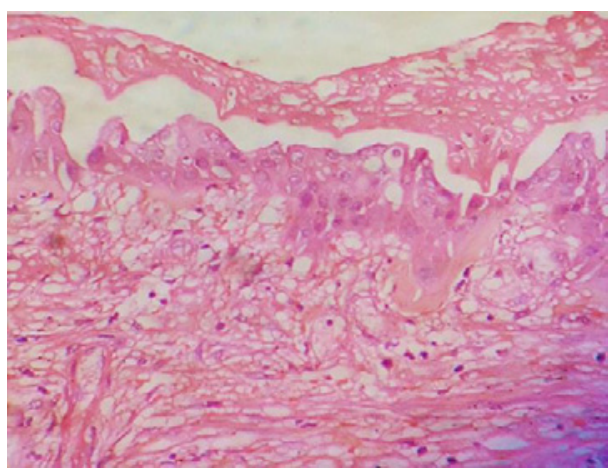

Figure 4: Histopathology showed features of Sialadenoma papilliferum.

specimen removed and sent for histopathological examination, which showed focally lobules of normal serous acing and many atrophic lobules infiltrated by lymphoid follicles and lymphocytic infiltrate, there was broad papillary projections seen in one of the cysts covered by cap of stratifies squamous epithelium with scattered mucus cells (Figure 4), hence the features were of Sialadenoma papilliferum of right parotid.

\section{Case 3}

Pleomorphic adenoma: A 66-year-old male came with complaints of right parotid swelling since 10years.On clinical examination, a firm; non-tender, nodular mass was palpated. There was no associated signs of facial nerve deficit noted. Based on clinical examination, signs and symptoms, a provisional diagnosis of pleomorphic adenoma was made. Fine needle aspiration biopsy and ultrasonography (well-defined solid and cystic lesion within deep lobe of right parotid) and CT scan (Figure 5C) suggestive of pleomorphic adenoma. Extra capsular dissection of parotid tumor done (Figure $5 \mathrm{~A}$ ) and specimen (Figure 5B) sent for histopathological examination and histopathology reported as pleomorphic adenoma of parotid salivary gland- encapsulated tumor tissue composed of alternating cellular and hyoid areas (Figure 6). 


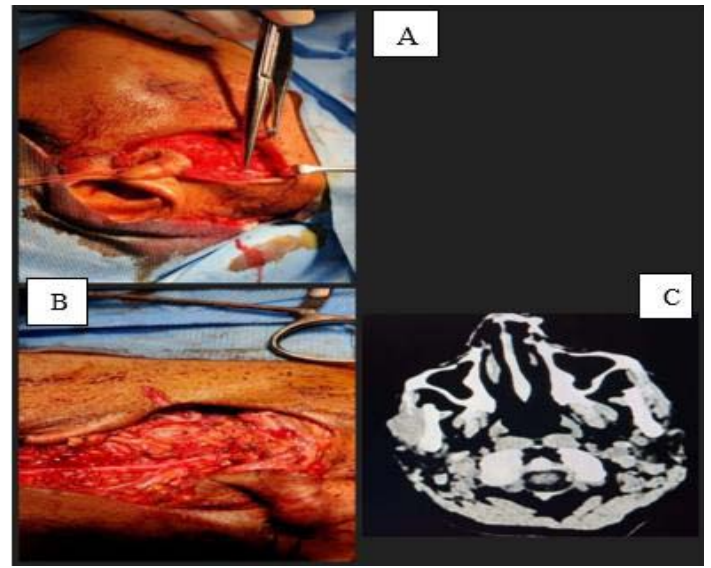

Figure 5: A) Extracapsular dissection of parotid tumour; B) Dissected specimen; C) CT scan suggestive of pleomorphic adenoma.

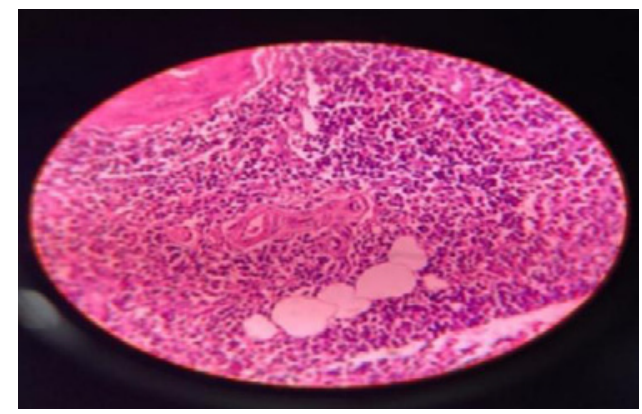

Figure 6: Histopathology revealed pleomorphic adenoma.

\section{Case 4}

Parotid basal cell adenoma: A 34-year-old female came with complaints of right side face/mandible swelling since 4 weeks. All necessary investigations were done. Ultrasonography of right cheek showed irregular hypo echoic lesion in anterior margin of right parotid gland. Computed tomography scan of face suggestive of right masseter muscle possibility of schwannoma/hemangioma (Figure 7C). Fine needle aspiration cytology suggestive of parotid basal cell adenoma. Incision placed and exposure done (Figure 7A). Extracapsular dissection of parotid tumor done (Figure 7B) and histopathology reported as chronic sialoadenitis-salivary gland acini with focal areas of dense fibrosis with entrapped acini, skeletal muscle fibers. Acute as well as chronic inflammatory cells noted lymph node with prominent germinal center seen (Figure 8).

\section{Case 5}

Parotid cystadenoma- keratin cyst: A 70-year-old male came with complaints of lump behind right side pinna since 0ne and half months. All necessary interventions were done. Computed tomography scan done and revealed ill-defined heterogeneously enhancing lesion in right parotid gland. Extra capsular dissection of parotid cyst adenoma- keratin cyst is done (Figure 9A) and sent for histopathological examination. Histopathology revealed parotid cyst adenoma-single large cyst lined by single to stratified layers of benign cuboidal to columnar cells forming papillary folds into the lumen. Lumen contains proteinaceous fluid granular epithelium lobules

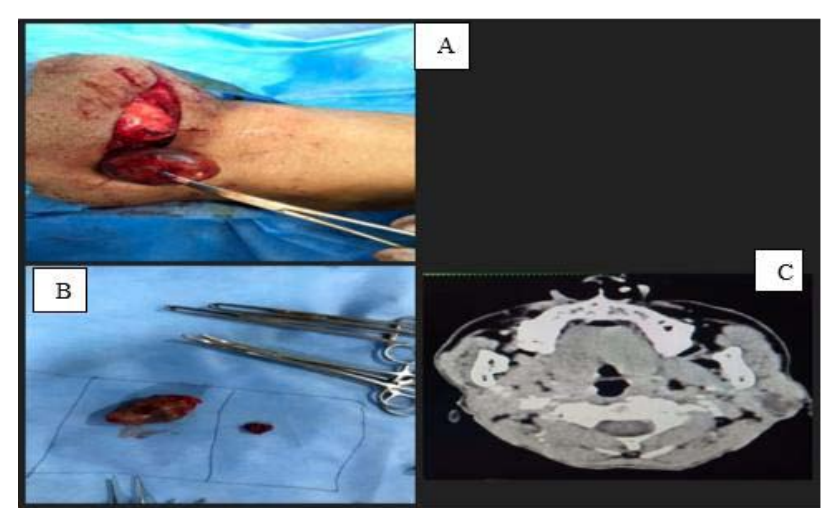

Figure 7: A) Incision and exposure; B) Extracapsular dissection of parotid tumours; C) Computed tomography scan of face suggestive of right masseter muscle possibility of schwannoma/hemangioma.

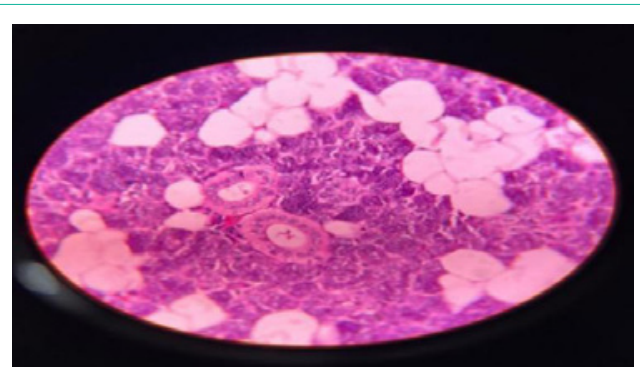

Figure 8: Histopathology suggestive of chronic sialoadenitis.

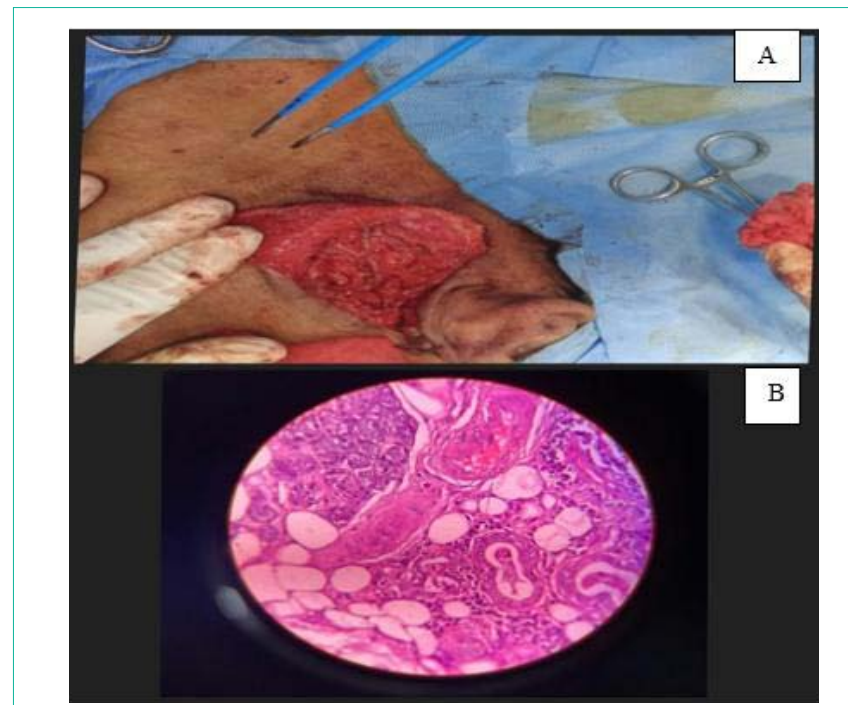

Figure 9: A) Extracapsular dissection of parotid cyst adenoma; B) Histopathology revealed parotid cystadenoma.

of cyst is surrounded by thick fibrous stroma with eosinophils and lymphocytes (Figure 9B).

\section{Discussion}

Salivary gland tumors are quiet uncommon complex neoplasms accounting for $2 \%-6.5 \%$ of the head-and-neck tumor. The incidence of the parotid tumor has increased in recent years [4].

Extra Capsular Dissection (ECD) differs markedly from classic 
surgical approaches to the parotid neoplasm because facial nerve dissection is not performed [1]. Surgery Intra operatively, the time of the operation starts with the induction of general anesthesia. The skin is marked, the flap is raised. The skin incision was performed while considering the natural folds of the face and neck flexion, and the incision starts (vertical segment anterior) from the pre-auricular region and extends up to the ear lobe insertion, reaches the anterior margin of the mastoid, and then continues posteriorly along the mandibular angle [6]. The skin incision (Blair) and the flap size may be adapted to the size and location of the tumor. After dissection of the subcutaneous tissue, the sternocleidomastoid muscle and the greater auricular nerve together with the capsule of the parotid gland are exposed. The parotid gland is rotated forward so exposing the posterior and deep surface of the gland as far as the posterior belly of the digastric muscle. This gives much improved access for ECD. A cruciate incision is then marked over the surface of the parotid lump extending approximately $1 \mathrm{~cm}$ peripheral to the tumor margin. After the tumor has been released from the surrounding tissue, the edges of the cruciate incision are re approximated and sutured together [1]. Follow-up After discharge from the hospital, the patients returned after one week for suture removal, with a weekly appointment for the first month, every two weeks for the second month, once monthly for the first year and as required after that.

\section{Conclusion}

In most traditional facial nerve dissection parotid procedures, the capsule of the parotid tumor is exposed during the operation without an increased risk of recurrence. Proper follow-up demonstrates that ECD in reported case series does not increase the incidence of recurrence. The advantages of ECD include the removal of the mass with adequate margins of healthy parotid tissue and a reduction in the side effects after surgery, thus preserving the parotid salivary function. A prolonged follow up is recommended [6]. Extracapsular dissection is a safe, reliable and recommended technique to manage the small benign superficial parotid tumors if the case is selected properly. The advantage is the reduction in transient nerve injury with ECD. Frey's syndrome, greater auricular nerve neuropathy and contour defects are minimized with ECD. As the needs for reducing morbidity and maintaining facial aesthetics increase, ECD represents the current limit of conservative parotid surgery. A common feature of all minimally invasive therapies is that the technique leaves little room for error [2].

Informed consent and patient details: The authors declare that this case series does not contain any personal information that could lead to the identification of the patients and/or volunteers. The patients understand that their names and initials will not be published and due efforts will be made to conceal their identity.

\section{References}

1. Robert LW, Heinrich Iro, Mark MG. The Role of Extracapsular Dissection for Benign Parotid Tumors Cur Otorhinolaryngology Rep. 2014; 2: 55-63.

2. McGuire M, Thomas BL and Renewal AG. Extracapsular dissection for clinically benign parotid lumps: reduced morbidity without oncological compromise. Correspondence: Professor M McGuire. British Journal of Cancer. 2003; 89: 1610-1613.

3. Micro S, Michael K, Miguel C, Konstantin's Mantsopoulos, Heinrich I. Extracapsular Dissection in Pleomorphic Adenomas of the Parotid Gland: Results After 13 Years of Follow-up. Laryngoscope. 2020: 1-7.

4. Sofa R, Safi SA. Assessment of the extracapsular dissection of the benign parotid tumors, extending the literature. S Riyadh, SS Abdulrazaq. Journal of Oral and Maxillofacial Surgery, Medicine, and Pathology. 2018; 30: 86-90.

5. Venkatesh S, Teerthanath S, Hariprasad S. Parotid Gland Tumors: 2 Year Prospective Clinicopathological Study. Annals of Maxillofacial Surgery. 2019; 9.

6. Maria GC, Eugenia A, Amerigo G, Walter C, Davide C, Ida B, et al. Pleomorphic Adenoma of the Parotid: Extracapsular Dissection Compared with Superficial Parotidectomy: A 10-Year Retrospective Cohort Study. Hindawi Publishing Corporation. The Scientific World Journal. 2014; 2014: 564053. 